\title{
Object recognition under semantic impairment: The effects of conceptual regularities on perceptual decisions
}

\author{
Timothy T. Rogers and John R. Hodges \\ MRC Cognition and Brain Sciences Unit, Cambridge, UK \\ Matthew A. Lambon Ralph \\ University of Manchester, Manchester, UK \\ Karalyn Patterson \\ MRC Cognition and Brain Sciences Unit, Cambridge, UK
}

\begin{abstract}
Although patients with semantic deficits can sometimes show good performance on tests of object decision, we present evidence that this pattern applies when nonsense-objects do not respect the regularities of the domain. In a newly designed test of object-decision, 20 patients with semantic dementia viewed line drawings of a real and chimeric animal side-by-side, and were asked to decide which was real. The real animal was either more typical (real $>$ nonreal) or less typical (nonreal $>$ real) than the chimera. Performance was significantly better in the real $>$ nonreal condition, and success in both conditions was modulated by patients' degree of semantic impairment. A similar effect of item typicality was revealed in a subset of items selected from a standard test battery. Object-decision scores were highly correlated with other pictorial and verbal assessments of conceptual knowledge, suggesting that impaired performance on all tasks resulted from the degradation of a unitary underlying system.
\end{abstract}

What is the relationship between perceptual knowledge and conceptual or semantic knowledge? How do the perceptual representations and processes that negotiate our experience with the environment give rise to our conceptual knowledge of it; and how in turn does conceptual knowledge influence perceptual recognition?

Requests for reprints should be addressed to Dr Timothy Rogers, Medical Research Council Cognition and Brain Sciences Unit, 15 Chaucer Road, Cambridge CB2 2EF, UK.

(C) 2003 Psychology Press Ltd

http://www.tandf.co.uk/journals/pp/01690965.html

DOI: $10.1080 / 01690960344000053$ 
Answers to these questions have tended to fall into one of two camps. The first follows a research tradition extending back to Lissauer (1890, cf. Humphreys \& Riddoch 1999) and heavily influenced by Marr (1982), in which a stimulus object or word must be recognised before its meaning can be accessed. "Recognition" in turn is construed as the process of matching a perceptual representation of the stimulus item to stored representations of previously encountered stimuli (or representations derived from these). Such stored "structural" representations are understood to be qualitatively different from the "semantic" representations that store meanings-they do not encode explicit semantic content, but capture information about visuo-spatial structure in the case of visual object recognition (see for example Biederman, 1987) or lexico-morphological structure in the case of word recognition (see Coltheart, Rastle, Perry, Langdon, \& Ziegler, 2001). For example, the dual-route cascaded (DRC) model of word-reading posits phonological and orthographic "lemma" representations that facilitate spoken- and written-word recognition, but which do not capture word meaning (Coltheart et al., 2001). Similarly, the interactive activation (IAC) model of object naming posits visual "structural descriptions" which permit the recognition of objects from vision prior to retrieval of explicit information about their functional and associative properties (Humphreys, Riddoch, \& Quinlan, 1988). Both models distinguish representations that encode explicit meanings from those that subserve stimulus recognition.

The second view also has a long provenance in cognitive science, extending at least back to Wernicke and other neuropsychologists of the late nineteenth century (see Eggert, 1977). Here, semantic knowledge does not reside in representations that are separate from those that subserve perception and recognition, but emerges from the learned associations amongst such representations in different modalities (e.g., Allport, 1985; Warrington \& McCarthy, 1987; Humphreys \& Forde, 2001). Neuroanatomically, the semantic system (on this view) consists in those cortical tracts and regions that permit the interaction of perceptual representations in different modalities, which need not be construed as capturing explicit, inherently meaningful semantic content (Rogers et al., in press; Rogers \& McClelland, in press; Rogers \& Plaut, 2002). Instead, meanings inhere only in the distributed patterns of activity provoked by a stimulus item or event across regions of cortex that are dedicated to the representation of modality-specific information: what things look like, how they move, the sounds they make, the words that describe them. This approach has found recent widespread endorsement in the interpretation of findings from functional neuroimaging (e.g., Chao, Haxby, \& Martin, 1999; Damasio, Grabowski, Tranel, \& Hichwa, 1996; Kellenbach, Brett, \& Patterson, 2001), EEG (e.g., Pulvermuller, 1999), and neuropsychology (e.g., Plaut, 2002), as well as in many contemporary theories of conceptual knowledge 
(e.g., Barsalou, this issue; Smith, 2000; Quinn, Johnson, Mareschal, Rakison, \& Younger, 2000; Rogers et al., in press).

The critical difference underlying the two approaches may be summarised thus: the former view differentiates between two kinds of representation-those that encode semantic content, or "meanings", and those that encode structural information sufficient to support stimulus recognition in a given modality of input. The second view does not distinguish between content-bearing semantic representations and contentfree structural representations, but emphasises that semantic memory serves a particular function-namely, the association in memory of modality-specific perceptual representations. Accordingly, we will refer to the former view as a content-based approach to semantics, and to the latter view as a process-based approach.

In the current paper we examine one line of evidence relevant to this debate from the study of object recognition, which has heretofore been taken to support content-based approaches to semantics: specifically, reports of cases in which brain-damaged patients appear able to recognise line-drawings of objects as familiar, despite being unable to retrieve semantic information about them. Such cases appear to provide some of the most convincing evidence that stimulus recognition is supported by a process that is functionally independent of semantic knowledge (Humphreys et al., 1988; Coltheart, Inglis, Michie, Bates, \& Budd, 1998). We will suggest an alternative explanation for the apparent preservation of visual object recognition under semantic impairment, more in keeping with a process-based approach to semantics, and will describe three experiments designed to test the explanation. The results suggest to us that the ability to recognise objects from vision always draws upon semantic resources, as required by process-based approaches, but that the consequences of disruption to the semantic system are only apparent in certain predictable stimulus conditions. In the general discussion we will consider implications of the current results for contemporary theories of semantic memory.

\section{PAST RESEARCH ON VISUAL OBJECT PROCESSING}

In the neuropsychological literature, the idea that visual object recognition is subserved by representations and processes that are functionally independent of semantics stems primarily from studies of object decision (Hillis \& Caramazza, 1995; Humphreys \& Riddoch, 1987, 1993; Rumiati \& Humphreys, 1997; Stewart, Parkin, \& Hunkin, 1992). In the typical experiment, participants are shown a series of single line drawings depicting either a real object, or a non-real object (a chimera) constructed from the parts of real items (e.g., a turtle's body with the head and neck 
of a snake). The participant's task is to accept the real objects and reject the chimeras. Because the individual parts from which the chimeric objects are built are all real (and familiar), and the chimeras themselves are as visually complex and well-formed as the real objects, accurate performance requires participants to make their judgements with reference to stored knowledge about whole familiar objects (Humphreys et al., 1988; Humphreys, Lamote, \& Lloyd-Jones, 1995). Object decision has become an important means of assessing the integrity of visual object recognition in the face of semantic and other kinds of neuropsychological disorders (Humphreys \& Forde, 2001; Rumiati \& Humphreys, 1997). When used together with tests of higher-level visual perception which do not draw on stored knowledge about real objects (such as the unusual-views matching test), and with tests of semantic memory, object decision is often interpreted as providing a tool for determining the locus of a visual processing impairment.

Take, for example, the seminal study of patient J.B. (Riddoch \& Humphreys, 1987) who, despite substantial impairments in retrieving semantic information from visual presentation of objects, performed within the normal range on "easy" and "difficult" tests of object decision (from the Birmingham Object Recognition Battery, or BORB; see Riddoch \& Humphreys, 1993). J.B. performed relatively well on tests of higher-level object perception (such as the view-matching task, which requires participants to decide which two of three photographs depict the same object from different viewpoints) and on purely verbal tests of semantic memory (such as naming to definition). Humphreys et al. (1988) concluded that J.B.'s difficulty did not lie in visual perception, visual object recognition, or semantics. Instead it resulted from damage to the tracts by which visual representations of known objects (structural descriptions) activate the functionally independent semantic representations that store meanings. Since this report, there have been a handful of similar cases (Hillis \& Caramazza, 1995; Sheridan \& Humphreys, 1993; Stewart et al., 1992), supporting the conclusion that visual object recognition does not depend upon intact communication with semantics, and must therefore be subserved by an independent recognition system.

Studies of patients with generalized semantic impairments complicate this picture to some extent. Particularly informative in this regard are studies of patients with semantic dementia: a neurodegenerative condition characterized by progressive atrophy of the anterior temporal lobes bilaterally (Garrard \& Hodges, 2000; Hodges, Garrard, \& Patterson, 1998). Patients with semantic dementia typically present with a moderate to severe anomia, accompanied by general impairment on a wide range of semantic tasks including category fluency, word to picture matching, sorting both words and pictures into conceptual categories, semantic 
matching tasks such as the Pyramids and Palm Trees test, concept definition to both words and pictures, sound-picture matching, demonstrating object use, drawing, and so on (e.g., Bozeat et al., 2003; Bozeat, Lambon Ralph, Patterson, \& Hodges, in press; Hodges, Graham, \& Patterson, 1995; Snowden, Goulding, \& Neary, 1989; Warrington, 1975). These deficits are not typically category- or modality-specific, and the degrees of impairment measured in different semantic tasks are typically highly correlated (Bozeat, Lambon Ralph, Patterson, Garrard, \& Hodges, 2000), although patients with predominantly left temporal atrophy often have greater difficulty with verbal relative to non-verbal semantic tasks (Lambon Ralph, McClelland, Patterson, Galton, \& Hodges, 2001).

Despite these often severe semantic impairments, other cognitive faculties appear to be remarkably spared well into the progression of the disorder. For example, speech remains grammatical and (apart from wordfinding difficulties) fluent, patients are well-oriented in time and place, and perform within the normal range on tests of episodic memory such as delayed recall of the Rey figure, and tests of mechanical problem-solving (Hodges et al., 1999a; Hodges, Spatt, \& Patterson, 1999b; Hodges, Bozeat, Lambon Ralph, Patterson, \& Spatt, 2000) and executive function (e.g., Wisconsin card-sorting, see Perry \& Hodges, 1999).

Patients with semantic dementia typically perform within the normal range on tests of visual object and spatial perception well into the disease progression. For example, they achieve normal scores in subtests of the Visual Object and Space Perception battery (VOSP; Warrington \& James, 1991); on tasks of matching the same object photographed from different views (see Hovius, Kellenbach, Graham, Hodges, \& Patterson, 2003); on tests of recognition memory for pictures of objects or faces (Graham, Simons, Pratt, Patterson, \& Hodges, 2000); and in the immediate and delayed conditions of the Rey figure copy (Hodges et al., 1999a,b).

Despite these spared abilities, the semantic impairment that characterises this syndrome can express itself in purely visual tasks that tap the participant's knowledge about real objects. For example, when patients are required to reproduce drawings of real objects from memory after a 10second delay, they show consistent, striking impairments. Increasingly with disease progression, they tend to omit the distinguishing and idiosyncratic visual properties of objects (such as the horns and udder on the cow); and to incorrectly add properties typical of a given category to atypical category members (e.g., adding four legs to a drawing of a seal; see Rogers et al., in press). The same patients are well able to copy the same drawings, so long as the stimulus remains present for them to consult-suggesting (together with their generally good performance on other visual tests) that the disorder spares visual object perception. Furthermore, the patients' relatively preserved scores on delayed-copying of non-meaningful stimuli 
(such as complex geometric shapes or the Rey figure) suggest visual, executive, and episodic memory resources that are sufficient to the copying task under delay conditions. Only when required to reproduce drawings of meaningful objects after a delay do they show substantial deficits (Bozeat et al., 2003).

The pattern of errors observed in delayed copying mirrors the general pattern of impaired performance observed across a range of different semantic tasks (Rogers et al., in press; Tyler, Moss, Durrant-Peatfield, \& Levy, 2000), including naming (Hodges et al., 1995), sorting, property verification (Warrington, 1975), word-picture matching (Funnell, 1996), and drawing to name (Lambon Ralph \& Howard, 2000). In all of these tasks, patients with semantic dementia demonstrate (a) a relative preservation of knowledge about properties that objects tend to share with their semantic neighbours, (b) rapid deterioration of knowledge about idiosyncratic or distinguishing object properties, (c) robust preservation of broad semantic distinctions (e.g., animal versus artifact) with declining sensitivity to narrower distinctions (e.g., pig versus goat), and (d) overextension of names and properties from typical category exemplars to their semantic neighbours (e.g., calling a pig a "dog"; drawing four legs on a picture of a seal). The pattern of errors observed in the delayed copy task neatly reflects these tendencies as well, suggesting that this task may be susceptible to the same underlying semantic impairment that disrupts performance on such clearly semantic tasks as naming, word-sorting, and word-picture matching.

The empirical data thus pose a certain puzzle. Studies of patients like J.B. suggest that the visual knowledge that supports visual object recognition is independent of semantic knowledge, as stipulated by content-based approaches to semantic memory (e.g., Humphreys et al., 1995; Coltheart et al., 1998). By contrast, studies of delayed copying in patients with semantic dementia suggest that impairments to semantic memory also compromise visual representations of meaningful objects, as required by process-based approaches to semantics.

The experiments we will describe here suggest a possible resolution to the apparent incongruity of these data. We suggest that the general robustness of knowledge about the shared and typical properties of semantically related objects may provide the basis for seemingly preserved performance in the object decision test under semantic impairment, owing to the fashion in which target and distractor stimuli are usually constructed. If the real-object targets in the test corpus consist mainly of items that share many visual attributes with their semantic neighbours, whereas most of the chimeric distractors include unusual and idiosyncratic visual features, patients may perform well simply by accepting typicallooking items and rejecting atypical-looking items. Put differently, the 
increasing restriction of their conceptual knowledge to general and typical features may lead the patients to accept any item whose properties are consistent with the regularities that are still relatively robust in the degraded system, and to reject or guess randomly on any unusual item whose properties violate those regularities.

Several previous studies of semantic dementia have included assessments of object decision, with discrepant outcomes ranging from virtually perfect performance (Lambon Ralph \& Howard, 2000) to scores no better than chance (Hodges, Patterson, \& Tyler, 1994). Of particular note, one case with an unusual category-specific pattern of semantic dementia was impaired at recognising natural kinds (especially fruits and vegetables), in line with his generally poorer semantic performance for natural kinds (Barbarotto, Capitani, Spinnler, \& Trivelli, 1995).

It is difficult, however, to draw any general conclusions regarding object decision performance in semantic dementia, because most reports so far are from single-case studies of patients with widely varying degrees of semantic deterioration and also tested with different sets of stimulus materials. The purpose of the present study was to obtain case-series data on object decision in semantic dementia, using materials designed to assess the impact of regularities in the real and non-real objects. In Experiment 1, we discuss a new object-decision test designed to measure the influence of conceptual regularities on object decision performance in a forced-choice paradigm. In Experiment 2, we investigate the influence of conceptual over-regularisation in the classic object-decision paradigm; and in Experiment 3 we consider the extent to which semantic task deficits and object decision deficits may be construed as arising from the same central semantic impairment in semantic dementia. The results of these experiments will then be discussed with reference to process- and content-based approaches to semantics.

\section{EXPERIMENT 1: THE OVER-REGULAR ANIMAL TEST (OAT)}

We tested object decision performance using a two-alternative forcedchoice design, in which the participants were shown line drawings of a real animal and a chimera side-by-side, and were asked to decide which was real. Half of the stimuli were constructed so that the chimera was effectively more prototypical than the real animal (the nonreal $>$ real condition) - for example, the patient might see a picture of a camel paired with a chimeric camel which had no hump. The remaining stimuli were constructed such that the chimera was atypical relative to the real animal (the real > nonreal condition) - for example, the patient might see a 
donkey paired with a chimeric donkey with a hump added to its back. By "typicality", we mean the propensity for an item to be composed predominantly of parts that are shared by familiar items in the same semantic category. For example, most familiar animals have tails and ears, whereas relatively few have humps on their backs or horns on their snouts. Thus, when shown a lion with or without a tail, and asked to decide which is real, the correct choice (the lion with a tail) is also the most typical stimulus. However when shown a gorilla with and without a tail, the correct choice (the gorilla without a tail) is the less typical stimulus. Sixteen such stimulus pairs were created in each condition, making 32 items in the test all together. Examples are shown in Figure 1. If object decision is supported purely by intact visual recognition processes, and if semantic dementia is a selective deficit of conceptual knowledge, then patients should be able to match the real-animal targets to stored visual representations, and should perform well in both conditions. On the other hand, if their visual recognition processes are contaminated by the overregularisation that results from degraded semantic processing, they should perform well in the real $>$ nonreal condition (in which chimeras are atypical relative to targets), but poorly in the nonreal $>$ real condition (in which chimeras are more prototypical than targets).

In this experiment, we employed only animal items, simply because the domain of animals has strong typicality structure, with many properties tending to be shared by well-known exemplars-artifact categories by contrast tend to have less apparent typicality structure (Garrard, Lambon Ralph, Hodges, \& Patterson, 2001; Tyler et al., 2000; Cree \& McRae, 2002; Rogers et al., in press). Patients with semantic dementia almost always show equivalent impairment for animal and artifact domains (Garrard,

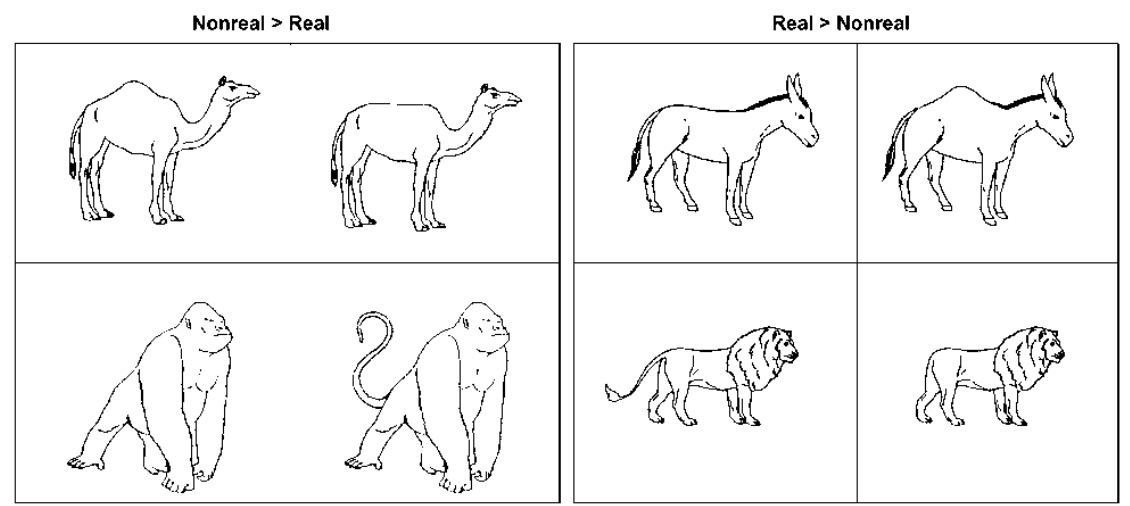

Figure 1. Examples of stimuli in the nonreal $>$ real and real $>$ nonreal conditions of the Over-regular Animal Test (OAT). 
Lambon Ralph, \& Hodges, 2002; Rogers \& Plaut, 2002), and we expect that the results obtained in the present experiment would extend well to artifact categories that have an equally strong degree of typicality structure.

To assess our manipulation of visual typicality, we asked 10 normal control subjects to judge the relative typicality of the visual features that differentiated real and chimeric stimuli in the OAT stimulus pairs. For example, participants were asked to judge whether the typical animal has a hump on its back or no hump on its back, long ears or short ears, four legs or two legs, etc. The volunteers were further instructed that in some cases the choice might be difficult to make, but that they should rely upon their own idea of what a "typical animal" might look like to make their decision. For all but four of the properties, participants unanimously judged as more typical the property that was consistent with our intended manipulationfor example, judging that the typical animal was unlikely to have a hump on its back, was likely to have a tail, was more likely to have four rather than two legs, etc. For the remaining properties (squat legs vs. long legs; short ears vs. long ears; straight neck vs. curved neck; crest on the head vs. no crest on the head) the majority of participants chose the property that was consistent with the intended manipulation.

\section{Methods}

Participants. Data were collected from 15 control participants from the MRC-CBU volunteer subject panel (age- and education-matched to the patient group) and from 20 patients with semantic dementia: 16 recruited through a Memory and Cognitive Disorders Clinic at Addenbrooke's Hospital, Cambridge; and 4 through the Neurology Department of the Royal United Hospital (RUH) in Bath, and the Research Institute for Care of the Elderly (RICE), St. Martin's Hospital in Bath. All patients matched the profile of semantic dementia that has been documented at length elsewhere (see Patterson \& Hodges, 2000, for a recent review). Scores on a range of standard neuropsychological tests (along with demographic data) are shown in Table 1. All patients were administered a semantic battery, and all showed mild to severe difficulty with picture naming, word-picture matching, and both verbal and visual variants of the Pyramids and Palm Trees test. In this and subsequent experiments, all data from a given individual were collected within a 6-month period. Scores on these tests are also shown in Table 1. In this and all subsequent tables and figures, patients are rank-ordered according to their scores on a 10alternative forced-choice word-picture matching task which provides a general index of the extent of their semantic impairment (Hodges et al., 1995). 
TABLE 1

Scores from 20 semantic dementia patients on a range of semantic and non-semantic neuropsychological tests

\begin{tabular}{|c|c|c|c|c|c|c|c|c|c|c|c|c|c|c|c|c|c|c|c|c|}
\hline Test & $\mathrm{AC}$ & WM & AN & $\mathrm{EO}$ & JP & $\mathrm{ATe}$ & MA & $\mathrm{JC}$ & DA & GO & SL & EK & AT & $\mathrm{KH}$ & GT & JG & DC & PD & $\mathrm{JH}$ & MK \\
\hline Age & 58 & 53 & 64 & 75 & 65 & 65 & 63 & 58 & 75 & 62 & 52 & 59 & 60 & 59 & 70 & 68 & 77 & 72 & 62 & 66 \\
\hline Education & 13 & 13 & 9 & 12 & 10 & 13 & 13 & 10 & 16 & 9 & 12 & & 10 & 9 & & 11 & 9 & & 10 & \\
\hline Sex & $\mathrm{M}$ & $\mathrm{F}$ & $\mathrm{M}$ & $\mathrm{F}$ & M & $\mathrm{M}$ & $\mathrm{M}$ & $\mathrm{M}$ & $\mathrm{M}$ & $\mathrm{M}$ & $\mathrm{F}$ & $\mathrm{F}$ & M & $\mathrm{M}$ & $\mathrm{F}$ & $\mathrm{F}$ & $\mathrm{F}$ & $\mathrm{F}$ & $\mathrm{F}$ & $\mathrm{F}$ \\
\hline \multicolumn{21}{|l|}{ Clinical } \\
\hline MMSE (/30) & 30 & 24 & 28 & 15 & 27 & 25 & 29 & 15 & 9 & 19 & 24 & 27 & 26 & 24 & 26 & 19 & 15 & 13 & 7 & 21 \\
\hline \multicolumn{21}{|l|}{ Rey } \\
\hline Copy & 36 & 36 & 36 & 25.5 & 36 & 36 & 36 & 31 & 34 & 34 & 30 & 34 & 23.5 & 36 & 34 & 34 & 29 & 36 & 34 & 30 \\
\hline Immediate recall & 24 & 23 & 29 & 0.5 & 24 & 23 & 17.5 & 8.5 & 16 & 26 & 16 & - & - & 12 & - & 3.5 & 3 & - & 5 & - \\
\hline Delayed recall & 23 & 25 & 27.5 & - & 24 & 24 & 6.5 & 8 & 17 & 18.5 & 14 & - & 3 & 12.5 & - & 4 & 4 & - & 0 & - \\
\hline \multicolumn{21}{|l|}{ Digit span } \\
\hline Forw & 8 & 8 & 8 & 6 & 5 & 8 & 6 & 7 & 3 & 6 & 5 & 6 & 5 & 4 & 6 & 6 & 5 & 7 & 6 & 5 \\
\hline Back & 7 & 5 & 6 & 3 & 4 & 4 & 3 & 3 & 2 & 3 & 4 & 7 & 4 & 3 & 4 & 4 & 3 & 5 & 5 & 4 \\
\hline \multicolumn{21}{|l|}{ VOSP } \\
\hline Screen $(/ 20)$ & 20 & 20 & - & 20 & 19 & 20 & - & 16 & 18 & 20 & 20 & 20 & 18 & 20 & 20 & 19 & 17 & 19 & 17 & 17 \\
\hline Incomplete letters $(/ 20)$ & 19 & 19 & 20 & 12 & 19 & 20 & 19 & 17 & 17 & 19 & 20 & 20 & 13 & 19 & 18 & 20 & - & 3 & - & 10 \\
\hline Dot count $(/ 10)$ & 10 & 10 & - & 10 & 10 & 10 & 10 & 8 & 9 & 10 & 9 & 10 & 10 & 10 & 10 & 10 & 10 & 10 & 10 & 10 \\
\hline Position discrimination (/20) & 20 & 11 & 20 & 17 & 20 & 20 & 20 & - & 19 & 20 & - & 20 & 16 & 19 & 20 & 20 & 18 & 16 & 19 & 17 \\
\hline Number location $(/ 10)$ & 10 & 10 & 10 & 5 & 10 & 9 & 10 & 2 & 10 & 10 & - & 10 & 3 & 10 & 10 & 10 & 10 & 9 & 10 & 6 \\
\hline Cube $(/ 10)$ & 10 & 10 & 10 & 1 & 10 & 10 & 10 & 10 & 8 & 10 & 10 & 10 & - & 10 & 10 & 10 & 4 & 5 & 9 & 6 \\
\hline Object decision $(/ 20)$ & 15 & 19 & 20 & 16 & 17 & 19 & 16 & 16 & 18 & 16 & 13 & 12 & 12 & 17 & 12 & 16 & 14 & 6 & 17 & 9 \\
\hline \multicolumn{21}{|l|}{ Semantic } \\
\hline WP-Match (/64) & 63 & 63 & 63 & 59 & 59 & 58 & 57 & 56 & 50 & 49 & 48 & 46 & 46 & 44 & 32 & 29 & 19 & 17 & 16 & 11 \\
\hline \multicolumn{21}{|l|}{ PPT (/52) } \\
\hline Words & 49 & 39 & 48 & 48 & 48 & 44 & 48 & 36 & 41 & 34 & 38 & 36 & 33 & 41 & 32 & 28 & 33 & 26 & - & 26 \\
\hline Pictures & 49 & 44 & - & 44 & 49 & 47 & 41 & 40 & 39 & 42 & 44 & 35 & 29 & 35 & 37 & 38 & 25 & 26 & 30 & 33 \\
\hline Naming (/64) & 50 & 57 & 62 & 30 & 57 & 10 & 13 & 33 & 18 & 7 & 18 & 17 & 20 & 30 & 11 & 6 & 3 & 4 & 5 & 2 \\
\hline
\end{tabular}

Note: Patients are ordered by their scores in the word-picture matching task. 
Procedure. Participants were told that they would see a series of picture pairs depicting similar-looking animals. They were instructed that the two pictures would differ in some important way, and that they must decide which picture showed a "real" animal. The participants were then shown the series of 32 test items in the same random order. For each item, the experimenter pointed out the difference between the real and chimeric animal, without referring to either the animal or the animal's parts by name. For example, the experimenter might say, "This one looks like this, and this one looks like this", while gesturing toward the feature that differentiated the real animal from the chimera. The participant was then prompted to decide which animal was real.

\section{Results}

Control participants performed well in both conditions of the task. In the real > nonreal condition, all controls performed perfectly. In the nonreal $>$ real condition, performance ranged from 14-16 correct (of 16). This may seem to indicate that the nonreal $>$ real condition was somewhat more difficult, but an item analysis revealed that all controls scored perfectly for all but two stimulus items in this condition. The two troublesome items were the raccoon and the gorilla, on which only about half of the control subjects made the correct choice. Dropping these two difficult items and their partners in the real $>$ nonreal condition from the analysis of patient data does not change the results of interest. In the following report of the results, the figures show the data from the entire set; but in our statistical analyses we will report results for both the complete set and the set with these items excluded.

The proportions correct in the two conditions are shown in Figure 2, for each individual patient and for the patient group as a whole. As noted earlier, the patients are ordered according to the magnitude of their semantic impairment as assessed by word-picture matching, with milder patients toward the left and more severe patients toward the right. Accuracy in both object-decision conditions declined to some extent with severity of semantic impairment, and significant correlations with the word-picture matching task were observed in both conditions $(r=.66, p<$ .001 for the real $>$ nonreal condition; $r=.77, p<.001$ for the nonreal $>$ real condition). For all except the mildest patients (whose scores were relatively good in both conditions), however, performance was worse for nonreal $>$ real items than for real $>$ nonreal pairs. For some cases, the discrepancy in performance is quite dramatic-for example, both A.T. and P.D. scored below chance for nonreal $>$ real items, but above $80 \%$ correct when the real item looked more typical. 


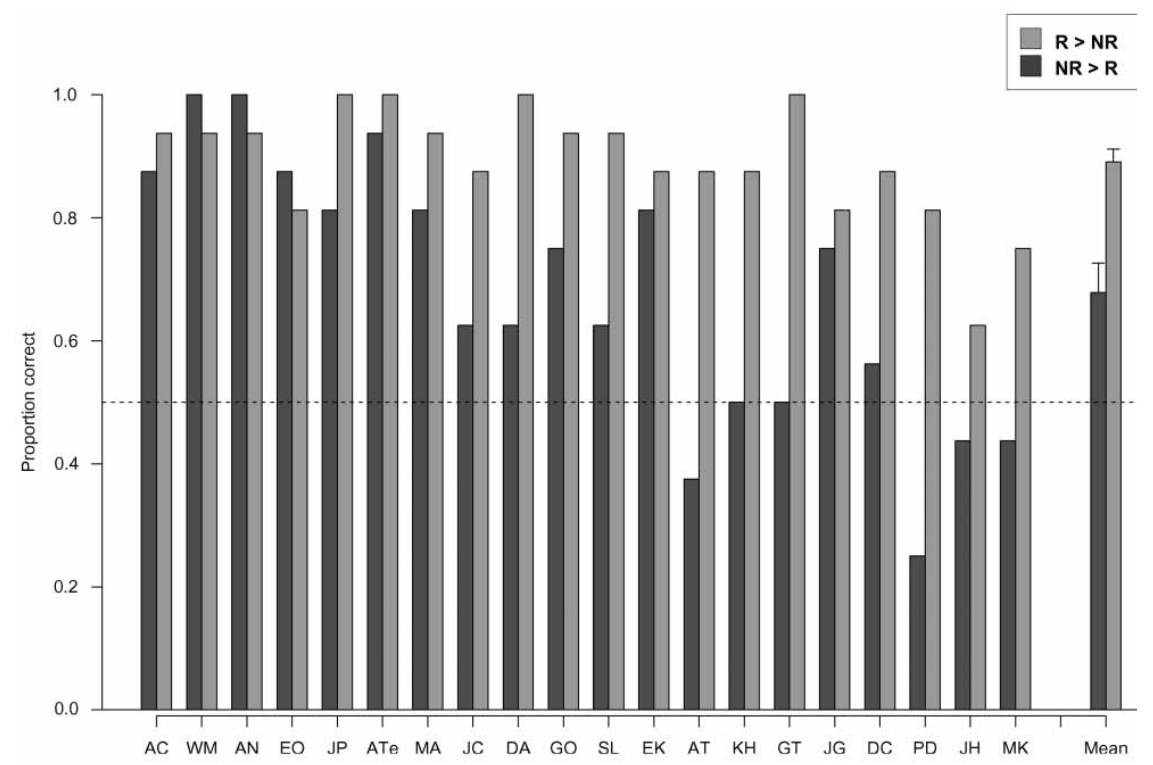

Figure 2. Proportion correct for nonreal $>$ real and real $>$ nonreal conditions of the OAT, for each of 20 semantic dementia patients, ordered by degree of semantic impairment. The dotted line indicates chance performance.

The data were subjected to an arcsine transformation and tested with a repeated-measures ANOVA using (transformed) proportion correct as the dependent measure, and item condition as the within-subject independent measure. The ANOVA confirmed that patients performed reliably better on real $>$ nonreal than nonreal $>$ real stimulus items, $F_{1}(1,19)=18.23, p$ $<.0001$ for the complete set; $F_{1}(1,19)=7.74 p<.02$ for the set with the two difficult items and their partners removed. The difference was also significant by items in both analyses, $F_{2}(1,30)=17.6, p<.001$ for analysis with all items; $F_{2}(1,27)=12.1, p<.003$ for analysis dropping difficult items.

\section{DISCUSSION}

Experiment 1, introducing a new test of object decision (the OAT), demonstrates that object decision performance in a forced-choice paradigm under semantic impairment is highly sensitive to the structure of the target and distractor stimuli. When real target objects (animals in this test) are typical-looking and chimeric distractors are atypical, object decision may seem to be spared even in patients with severe semantic deficits. For example, P.D. scored correctly on only 17 of 64 items $(27 \%$ correct) in the word-picture matching task, but chose correctly for 13 of 16 
items $(82 \%$ correct) in the real $>$ nonreal condition of the OAT. When target objects are unusual and chimeras are typical-looking, however, the picture changes dramatically: even patients with relatively mild semantic deficits may perform poorly. For example, J.C. exhibited a mild semantic impairment in the word-picture matching task (56/64, or $87 \%$ correct), but chose correctly for only 10 of 16 nonreal > real items in the OAT $(67 \%$ correct). This difference is particularly striking when one considers that chance performance is $10 \%$ in the word-picture matching task, but $50 \%$ in the object decision task. Considering nonreal $>$ real items in isolation, patients like J.C. may seem to have an object-decision deficit with only mildly degraded semantic knowledge; and considering real $>$ nonreal items alone, patients like P.D. may seem to have spared object recognition with substantially degraded semantic knowledge. Considering the two conditions together, we can see that these differences are a factor of the stimulus properties, and not of the neuropsychological profiles of the two patients.

One of the patients (J.G.) seemed to perform disproportionately well in the nonreal > real condition of the OAT, given the degree of her semantic impairment. From comments made during the test session, it was clear that one of J.G.'s correct choices in this condition, and at least two of her choices in the real $>$ nonreal condition, arose from her confusion about the identity of the different animal parts. When shown pictures of a normal horse and a chimeric horse with an udder added, J.G. chose the chimera and laughingly said, "It's a horse, isn't it—a male horse". Apparently she had mistaken the udder for male genitalia. When shown the matched stimulus in the alternate condition (a cow with and without an udder), she again picked the animal with an udder (this time correctly), and again commented that it must be a male. Finally, when shown the deer with four or with six legs, she incorrectly chose the chimera, but commented that it "had too many legs". Since the real and chimeric items differed only in the number of legs, it is not clear on what basis J.G. made her decision. The discrepancy in performance between the two conditions was small in J.G.'s case; but in light of these comments it is difficult to conclude that J.G. succeeded in object recognition. Nevertheless we will consider J.G.'s object decision performance in further detail in Experiment 2, to determine whether she constitutes a counter-example to our claim that semantic impairment necessarily compromises visual object recognition.

Three further points are of interest. First, the patients' poor performance in the nonreal $>$ real condition cannot be attributed to any deficit of episodic memory. In past work, it has been difficult to rule out the possibility, however unlikely, that deficits observed in the delayed-copy task actually reflect a subtle episodic memory impairment in semantic dementia. However, the current task does not require participants to retain 
visual information in memory over a delay, and in any case the demands of the two conditions are identical-hence it is difficult to explain the data with reference to episodic memory problems.

Second, because each stimulus item consists of a real and a chimeric animal side-by-side, participants were always faced with a correct option when making their decision in the current experiment. It therefore seems an inescapable conclusion that the patients were failing at recognition. If the patients had intact "structural descriptions", and if the processes that activate such representations were functionally independent of semantics, the patients should have recognised the real animal target in each stimulus pair. The results thus suggest either that visual object recognition processes are not independent of semantics, or that the non-semantic visual representations and processes that support recognition are conjointly impaired along with semantic memory in these cases. We will return to this issue in Experiment 3.

Finally, the discrepancy in performance between the two conditions is consistent with the account of spared object-decision in the face of semantic impairment that we sketched out in the introduction. The more impaired patients in Experiment 1 were likely to accept prototypicallooking animals and reject unusual-looking animals, regardless of whether they were real. The finding suggests that patients with semantic impairments will seem to have intact object recognition in the standard object-decision procedure if real targets are more prototypical than chimeric distractors. We investigate this possibility further in the next experiment by comparing patient performance on the OAT with performance on the BORB.

\section{COMPARING THE OAT AND THE BORB}

We designed Experiment 2 to fulfil two aims. First, although results from the OAT are strongly suggestive, it is difficult to know how the data from this task relate to object decision performance in the standard single-item yes/no procedure. In contrast to the usual task, the forced-choice procedure required participants to direct their attention to two different stimuli, to select one as a suitable target for the response, and to refrain from acting on the other. It is possible that these differences tax executive and attentional systems in ways that the standard procedure does not. It is therefore useful to evaluate the degree of impairment evident in the two conditions of the OAT with reference to well-known tests of object decision such as the BORB. Thus in Experiment 2a, we compare performance on the OAT and the short version of the BORB employed by Riddoch and Humphreys (1993) for 13 of the 20 semantic dementia patients described above. 
Second, we were interested to know whether the regularity effects witnessed in Experiment 1 might also be observed in the standard objectdecision procedure. To this end, we collected data on the long version of the BORB from four of our participants in Experiment 2b. From this battery we culled a subset of very prototypical and very atypical real animals and chimeric distractors, and assessed performance on these as a function of typicality and stimulus type (real or chimeric).

\section{Experiment 2a: Comparing the magnitude of impairment in BORB and OAT}

Procedure. Nine of the 20 semantic dementia patients described above were tested on the short version of the BORB object decision task, and four patients were tested on the complete version. The long form of the task consists of 128 line drawings, half depicting real objects and half chimeric objects matched for visual complexity. Though most of the items in the BORB object decision task are animals, this battery also includes a set of artifact items. The BORB is administered in four blocks of 32 items each (half real and half chimeric). Two blocks include relatively easy stimuli (as judged by university undergraduates) and two contain somewhat harder items. The short version consists of one easy block and one hard block from the complete test. In this experiment we will consider the performance of all 13 patients as a group on the items from the short version of the task.

The usual object decision paradigm employed in the BORB proceeds as follows. Patients are instructed that they will see a series of line drawings, some depicting real objects and some not. For each item, the participants are to indicate whether or not they think it is real. Items are presented in the same randomly generated order for all participants.

\section{Results}

The left-hand panel of Figure 3 displays the means and standard errors of the proportion correct for all 13 patients on the short 64-item BORB object decision and on the two conditions of the OAT from Experiment 1. Performance on the BORB task fell mid-way between the two conditions of the OAT. Average performance was also reflected in the individual data: proportion correct on the BORB fell between performance on the two conditions of the OAT for 11 of 13 patients. For the two exceptions (E.K. and J.G.) performance on the BORB was worse than performance on either of the OAT conditions.

The middle and right-hand panels of Figure 3 shows the same data calculated separately for milder and more severe patients, as determined by a median split on word-picture matching scores across the 13 patients. 

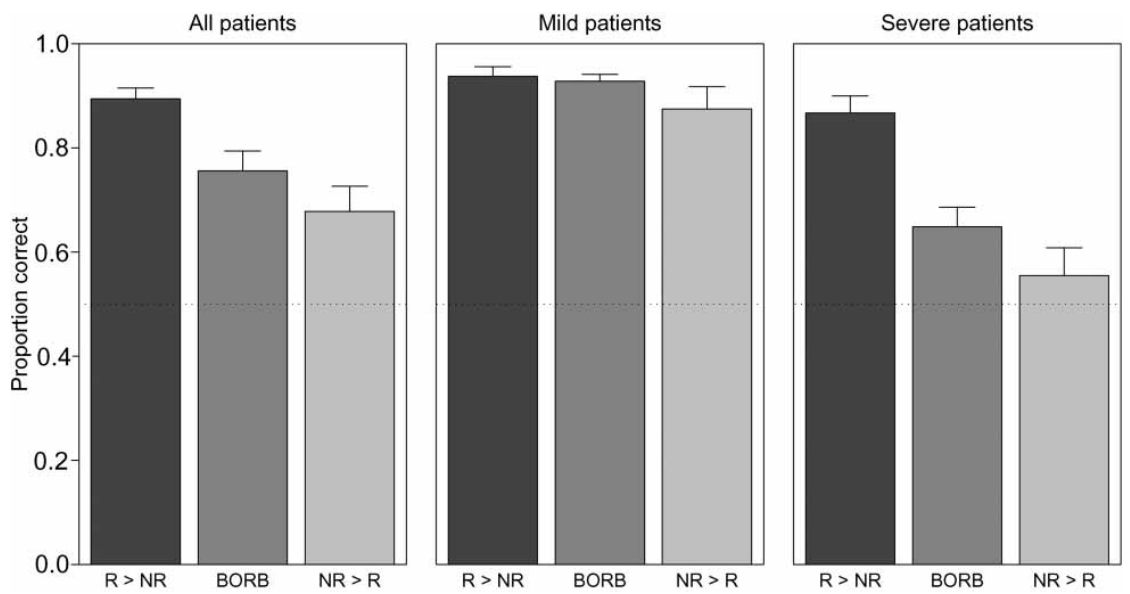

Figure 3. Mean proportion correct for real $>$ nonreal OAT $(R>N R)$, the short version of the BORB object decision, and nonreal $>$ real OAT $(N R>R)$ for all patients (left panel), and separately for milder (middle) and more severe (right) patients, as measured by a median split on word-picture matching. Error bars indicate the standard error of the mean for the different factor levels.

The pattern described above is clearly influenced by the severity of semantic deficit. Milder patients (scoring $75 \%$ or better on the wordpicture matching test) performed well in all three tasks, with only small differences apparent across them (although in the predicted direction). The performance of the more severe patients (scoring less than $75 \%$ correct on the word-picture matching test), by contrast, varied substantially across the three conditions, with the nonreal $>$ real condition of the OAT near chance, the BORB somewhat better, and the real $>$ nonreal condition of the OAT yielding better than $80 \%$ correct. The data from the more severe group demonstrate that the very same set of semantically impaired patients may show object-decision scores that do not differ from chance (nonreal > real OAT); are better than chance but substantially off ceiling (BORB); or are only a little off ceiling (real > nonreal OAT). Whether the patients appear to have a deficit of object recognition thus depends upon which test is used.

We tested the statistical reliability of these observations with a repeatedmeasures analysis of covariance, in which testing condition (nonreal $>$ real OAT, BORB, or real > nonreal OAT) was treated as a within-subject factor, and severity of impairment as assessed by the word-picture matching task was treated as a between-subjects covariate. Scores on the different object-decision tasks were converted to proportions and subjected to an arcsine transformation prior to conducting the ANCOVA. The results revealed significant main effects of both severity of impair- 
ment, between-subjects effect $F(1,11)=20.86, p<.001$ for all data; $F(1,11)=7.86, p<.001$ for the reduced OAT set with difficult items removed, and test condition, within-subjects effect $F(2,22)=9.33, p<$ $.001 ; F(2,22)=18.98, p<.001$ for the reduced OAT set. Post-hoc contrasts of the condition effect revealed that, across all patients, performance on the real $>$ nonreal condition was reliably better than performance on the other two conditions $(p<.01$ in full and reduced OAT sets), but that performance on the BORB was not significantly better than on the nonreal $>$ real condition of the OAT $(p=n . s$. in both OAT sets). The interaction of these factors was also significant, $F(2,22)=3.86$, $p<.04$ for all data; $F(2,22)=6.07, p<.04$ for reduced OAT set, indicating that the differences among test conditions were greater for the severe patients than for the milder patients, as Figure 3 suggests.

To determine how scores for milder and more substantially impaired patients compare with chance performance, we divided the 13 patients into "mild" and "severe" groups using a median split on their word-picture matching scores, and calculated $95 \%$ confidence intervals on the transformed mean proportion correct for all three object-decision measures. These confidence intervals were then reverse-transformed to derive appropriate intervals in the original proportion-correct measure. Amongst the mild patients, the confidence intervals for the three test conditions overlapped: for the nonreal $>$ real OAT the estimate ranged from 0.71-0.95; for the BORB, from 0.87-0.95; and for the real > nonreal OAT, from 0.91-0.96. Amongst the severe patients, the estimate for the nonreal > real OAT ranged from $0.42-0.67$, meaning that severe patients did not perform reliably better than chance in this condition. For the BORB, the $95 \%$ confidence interval ranged from $0.56-0.69$ which is reliably better than chance, even though not reliably better than performance in the nonreal $>$ real condition of the OAT. Finally, in the real $>$ nonreal condition of the OAT the confidence interval for severe patients ranged from $0.81-0.91$, reliably better than both other conditions.

Finally, we note that J.G., the patient who performed unusually well in the nonreal $>$ real condition of the OAT, chose correctly on only $63 \%$ of the items in the BORB. The two patients closest to J.G. in the extent of their semantic impairment (G.T. and D.C.) scored 67 and $66 \%$ correct, respectively. Thus there is no indication from the BORB that J.G.'s object recognition is disproportionately spared given the degree of her semantic impairment.

Comment. The results of Experiment 2a show that performance in the standard object decision task can be compromised under semantic impairment, just as is performance on the OAT. Moreover, the magnitude of the deficits on the short version of the BORB is just what one might 
expect from these patients' OAT scores, assuming that BORB stimuli were selected without regard for the degree to which targets and distractors are typical-looking. One can view the two subsets of the OAT as exemplifying polar stimulus conditions: one in which targets are always more typical than distractors, and one in which the reverse is true. If object decision deficits arise primarily from sensitivity to typicality in semantic dementia, these conditions should represent the upper and lower bounds of performance under semantic impairment, as they appear to do. Because items in the BORB were not designed to manipulate typicality, performance on this task should fall somewhere between these bounds, as indeed it did both in the average patient data and in all but two of the individual cases.

\section{Experiment 2b: Over-regularisation in the BORB}

Is there more direct evidence that a preference for typicality in semantic dementia can influence performance in the standard object decision task? To answer this question, we examined the performance of four patients (E.K., P.D., G.T., and M.K.) on a subset of real and chimeric items from the BORB, selected to be either highly typical-looking, or highly atypical. The four patients considered were those recruited in Bath, who were tested on the full complement of items in the long version of the BORB (whereas the remaining patients from Cambridge completed just the 64 items in the shorter version). As indicated in Table 1, all four fall in the severe end of the disease spectrum.

Procedure. We examined the 128 stimulus items from the full complement of the BORB object decision test, initially searching for chimeric stimuli that had the typical properties of four-legged land animals. We counted as "typical" those chimeras that met the following criteria. First, they had to share the properties of animals that are preserved in delayed-copy and drawing tasks by patients with semantic dementia: four legs, a body, a tail, a head, and eyes. Second, they could not include parts from animals spanning grossly different animal categories; for example, we rejected chimeras made from mammal and bird parts. Third, they had few distinguishing or idiosyncratic features. Of the 64 chimeric stimuli in the BORB, 11 met these criteria-7 from the "hard" stimulus set, and 4 from the "easy" stimulus set. We next selected 11 typical-looking real animal drawings ( 7 from the hard and 4 from the easy set) to match these, using the same criteria for selection and avoiding the high-frequency items cat, dog, and horse.

We then selected 11 atypical chimeras and 11 atypical real animals from the BORB, choosing 7 from the hard set and 4 from the easy set in each 
case. By "atypical", we mean items that violated the constraints described above: animals with idiosyncratic features, or animals without tails, four legs, etc. Thus these 44 items in the BORB constitute a subset in which two factors-real or chimeric, and typical or atypical-are fully crossed; and within each cell, difficulty is approximately matched.

\section{RESULTS}

Figure 4 shows the mean proportion correct across patients in each factor cell, as well as the data for each patient individually. Amongst the typicallooking items, patients were near ceiling for the real targets and near floor for the chimeric distractors. In other words, when confronted with a typical-looking stimulus, whether real or chimeric, patients usually judged that it was a real animal - thereby scoring correctly for real items and incorrectly for non-real items. By contrast, amongst the atypical items, patients behaved somewhat more randomly for both real and chimeric stimuli.

These observations were confirmed by statistical analysis in a withinsubjects ANOVA treating proportion correct for each stimulus condition as the dependent measure, and typicality (high or low) and stimulus type (real or chimeric) as within-subjects factors. As Figure 4 would lead one to expect, there was no significant main effect of typicality: participants were effectively at chance overall for both typical and atypical stimuli (both by subjects and by items, $F<1$ ). Patients tended to be more accurate when the item was real than when it was chimeric, $F_{1}(1,3)=6.1, p<.1 ; F_{2}(1$, $40)=22.8, p<.001$. As the figure shows, however, this effect is entirely due to the fact that the patients scored so well for real items and so poorly for chimeras in the typical condition, by virtue of almost always deciding that typical-looking items were real. Accordingly, the interaction between stimulus type and typicality was reliable by subjects and by items, even

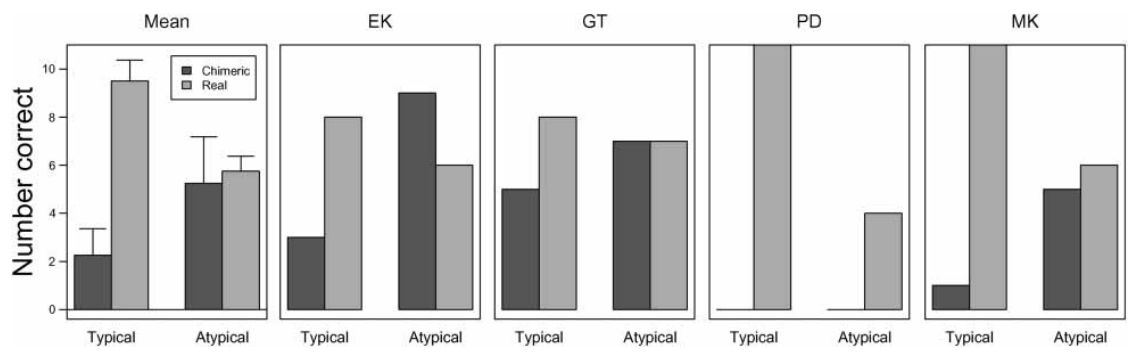

Figure 4. Performance on the subset of typical and atypical BORB stimuli averaged across four semantic dementia patients. Error bars indicate standard error of the mean in each condition. 
with this small number of participants, $F_{1}(1,3)=26.3, p<.02 ; F_{2}(1,40)=$ $17.4, p<.001$. On average, patients tended to accept typical-looking stimuli as real, regardless of whether or not they were; and when confronted with an unusual-looking animal, patients guessed at random for both real and chimeric stimuli.

The interaction of interest is apparent by inspection in each individual patient's data - in all four cases the difference in performance between real and chimeric items was larger (and positive) for typical stimuli than for atypical stimuli. The interaction is even apparent in patient P.D., who was strongly biased to accept most stimuli (rejecting only a handful of atypical-looking real animals). An analysis of log odds ratios revealed that this interaction was statistically significant for two of the four individuals (E.K., $z=2.28, p<.01$, and M.K., $z=1.98, p<.05$ ). It should be noted that with only 11 observations in each cell, there is little power to detect significant interactions in individual patients-the reliable effects observed in E.K. and M.K. reflect the large magnitude of the effect in these cases. From the graphs in Figure 4, it is apparent that the significant effect revealed in the group analysis is not carried solely by E.K. and M.K.- the interaction is in the same direction in all four cases, which is why the ANOVA yields a significant result, despite the small sample size.

Comment. Experiment $2 \mathrm{~b}$ provides good evidence that the behaviour of semantically impaired patients in the object decision task is strongly influenced by the typicality of the target and distractor stimulus items used in the BORB. Patients with severe semantic deficits accepted typicallooking items as real, and guessed randomly in response to atypicallooking items. If typicality is controlled in the stimulus set, this pattern of behaviour will yield chance performance, as it did for four patients across the subset of items we examined. However, chance performance may not be observed even for such severe patients when stimulus items in the test are not balanced in this way. If most of the real animals in the BORB are typical-looking, semantically impaired patients will be likely to score correctly for these items. If most of the chimeras in the test are atypicallooking, patients guessing randomly will choose correctly for half of these stimuli. Across such an unbalanced set, then, patients who are completely unable to discriminate real from chimeric animals when typicality is controlled may be expected to exceed chance in the test, as did the four severely semantically impaired patients described here.

A second point of interest is the finding that the four patients in Experiment $2 \mathrm{~b}$ did not consistently reject atypical items, but instead seemed to guess randomly when faced with an unusual-looking stimulus. That is, the patients were willing to accept most typical-looking stimuli (including chimeras) as real; but were not willing to reject most atypical- 
looking items as nonreal. Why was typicality almost uniformly alluring, when atypicality was not uniformly off-putting?

One possibility is that the stimulus items in the matched subset did not perfectly capitalise on the visual regularities that constrain impaired performance in the task. Though past studies of drawing to name and delayed copying suggest that visual attributes common to items in the semantic domain are more likely to be preserved than are more idiosyncratic properties, it is difficult to know precisely which aspects of visual object knowledge will prove robust to substantial semantic impairment. Perhaps the visual structure of some proportion of both real and chimeric stimulus items in the "atypical" subset was sufficient to permit chance performance on this set overall (as opposed to belowchance performance for atypical real animals, and above-chance performance for atypical chimeras).

A more interesting possibility is that this pattern reflects some spared knowledge about the potential for variability within the domain of animals. Though the patients clearly have degraded knowledge about the visual appearance of particular unusual-looking animals, they may retain the more general knowledge that it is possible for animals to deviate substantially from the visual prototype that characterises their state of knowledge about the domain generally. On this view, patients feel confident that typical-looking animals are likely to be real, because such stimuli conform to the knowledge that they retain about animals. Unusuallooking animals do not conform to this robust knowledge, and hence do not offer tempting targets; but patients guess randomly for these items, because they remember that there are some unusual-looking real animals in the world, even if they do not know whether the item facing them is one of these.

\section{COMPARING OBJECT DECISION AND SEMANTIC TASKS}

We have demonstrated that patients with semantic dementia show consistent deficits in object decision, which mirror the pattern of semantic impairment revealed in other tasks. Specifically, as conceptual knowledge deteriorates, patients increasingly tend to accept (as "real") objects constituted of parts that are robust to semantic impairment-those that are typical of a semantic category or domain — and to reject or guess randomly when faced with items that violate such regularities. These data are at least consistent with the view that the processes supporting visual object recognition and those supporting semantic memory are highly interactive.

It is difficult, however, to rule out the possibility that the observed deficits in object decision arise, not solely from impairment to a unitary 
and interactive semantic system, but from the conjoint impairment of functionally independent semantic and visual recognition systems. The patients we have discussed perform quite well on tests of visual perception, such as the non-semantic subtests of the VOSP and the immediate copying of abstract and real-object stimuli. However, these tests do not eliminate the possibility that there exists an intermediate level of visual processing, functionally independent of earlier vision and later semantics, which corresponds to a stage of pre-semantic visual recognition and which is gradually compromised in semantic dementia.

In Experiment 3 we sought further evidence for our preferred hypothesis, by examining the degree to which performance on various uncontroversially semantic tasks covaries with performance on different forms of object-decision. Consider what one might expect to find if visual object recognition and semantic processes are functionally independent but conjointly impaired. Across individuals, the degree to which each independent system is compromised by the disease process will likely vary to some small extent, even if both systems are affected together from the beginning. In this case, the magnitude of the correlation between tasks that tap object-recognition processes on the one hand and those that tap semantics on the other may be high; but it should not be as high as the degree of intercorrelation within various different semantic tasks, or various different object-recognition tasks. On the other hand, if objectrecognition deficits and semantic deficits result from the same central impairment, we might expect the correlations between the two kinds of tasks to be as great as the intercorrelations among different versions of the same kind of task. The goal of Experiment 3 was to measure the extent to which scores on different object decision tasks correlate with one another, with other semantic tasks that tap visual and/or verbal semantic knowledge, and with other non-semantic tasks.

\section{Method}

The 20 semantic dementia patients described in Experiment 1 were tested on a further well-known object-decision task (the object-decision component of the VOSP), so that, together with the data from Experiments 1 and 2, we were able to examine scores on four varieties of object decision (the two conditions of the OAT, the BORB, and the VOSP). The same patients were also tested on five semantic and eight nonsemantic tasks.

The semantic tasks varied in the extent to which they required intact visual recognition and verbal comprehension skills. They were as follows.

Pyramids and Palm Trees Test. A semantic matching task in which participants are required to match one of two candidate response items to 
a sample stimulus on the basis of semantic relatedness (Howard \& Patterson, 1992). For example, the participant is shown a picture of a pyramid as the sample, and asked to decide which of a pine tree or a palm tree best matches it. The test consists of 52 items and may be administered with either picture or word triads. We conducted both versions for purposes of comparison to object decision. Note that the picture version requires intact semantics and visual object recognition, but does not require verbal comprehension; whereas the word version requires verbal comprehension, but does not tap visual object recognition.

Camel and Cactus Test. A test designed on the same principle as the Pyramids and Palm Trees Test above, but with a larger number of items (64), and with four response choices rather than two. It may also be administered with pictures or words as stimuli; both are reported here. See Bozeat et al. (2000) for further detail.

Word-Picture Matching. This is a 10-alternative forced-choice wordpicture matching task in which the participant is given the spoken name of an object and is asked to which of 10 real-object line drawings (1 target and 9 foils) it refers. Sixty-four target stimuli were drawn from six semantic categories, half living things and half non-living things (land animals, birds, fruits, household objects, vehicles, or tools). Distractors were drawn from the same category as the target (see Bozeat et al., 2000, for further details). The word-picture matching task requires intact verbal comprehension, semantics, and visual object recognition.

The eight non-semantic tasks consisted of three tasks that draw upon frontal/executive resources (the Rey figure copy, forward digit span, and backward digit span); and five subcomponents of the VOSP designed to test visual perception (dot counting, position discrimination, incomplete letters, cube drawing, and number location; see Warrington \& James, 1991).

Most patients were able to complete most tasks, but we were unable to obtain data from all patients for 5 of the 17 tasks. Each patient completed the tests within an 8-month time window. To determine how performance covaried across the various tests, we first considered the pairwise correlation coefficients for all pairs of tests. We then conducted a confirmatory factor analysis to determine how best to explain the observed patterns of covariation.

\section{Results: Analysis 1}

The left-most panel of Figure 5, labelled "Within task", shows the correlation coefficients for all pairs of object-decision tests ("Obj Dec"), all pairs of tasks requiring visual object recognition and semantics ("Vis- 


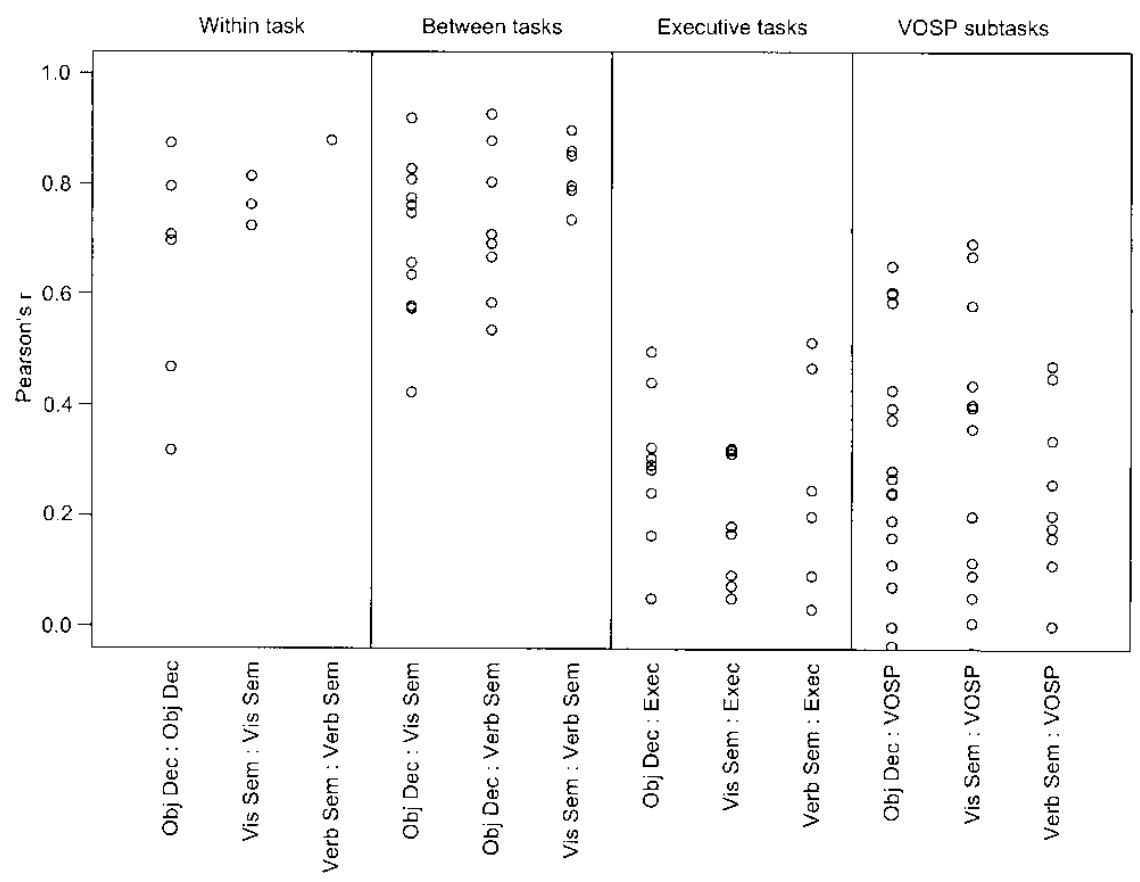

Figure 5. Correlation coefficients for pairs of similar tasks (left-most panel); between objectdecision, visual, and verbal semantic tasks (second from left panel); and between these tasks and frontal/executive tasks (second from right) and tests of visual perception (rightmost panel).

Sem"), and the two verbal-semantic tests ("Verb-Sem"). The next panel (labelled "Between task") reflects the correlation coefficients for all pairings of tests between these groupings. As the illustration makes clear, the degree of association is of comparable magnitude for between-task comparisons and within-task comparisons. Two of the three lowest correlation coefficients occurred between different varieties of object decision (the correlation between the two conditions of the OAT, and between real > nonreal OAT and the VOSP); and two of the four highest correlation coefficients observed were between an object decision task (the BORB) and the two purely verbal semantic tasks (the word-only versions of the Camel and Cactus test and the Pyramids and Palm Trees test; $r=.88$ and .93 respectively).

The two right-hand panels in Figure 5 show how the object-decision, visual-semantic, and verbal-semantic tasks correlate with the tasks that draw upon frontal-executive resources (second panel from right) or visual perception (rightmost panel). Here correlations are relatively low, and importantly, the semantic and object-decision tasks show similar degrees 
of association with the sets of non-semantic tasks. The results indicate that the impairments in semantic and object-decision tasks, and the high degree of association among these, do not result from a global deficit that affects all cognitive domains.

\section{Analysis 2}

The relatively small number of cases over which these correlation coefficients were calculated (ranging from 10 to 20), as well as the fairly large number of potential comparisons, limit the power to detect reliable differences among the various correlation coefficients. Our hypothesis stipulates that the magnitude of correlation within object-decision and within semantic tests should be no greater than the degree of correlation between these types of tests; hence this lack of statistical power poses a particular difficulty in testing our hypothesis.

In the second analysis we addressed this issue by conducting a confirmatory factor analysis designed to contrast three different models of the factors underlying object decision and semantic task performance in our patient sample, using a subset of the observed variables. The relatively small sample size is also a serious concern in this case; however, by restricting the number of observed measures to 5 we found that we were able to fit the various models and come near to the statistical rule of thumb advocated by Bryant and Yarnold (1995), which states that the ratio of subjects to variables should not fall below 5 .

From the object-decision and semantic tasks listed above, we employed five observed measures in the factor analysis: the proportion correct for the complete OAT (i.e., the sum of nonreal $>$ real and real $>$ nonreal conditions divided by 32), the VOSP object decision task, word-picture matching, Pyramids and Palm Trees with pictures, and Pyramids and Palm Trees with words. Of these five measures, two require object-recognition alone (OAT and VOSP), two require object-recognition as well as semantic capabilities (WP-Match and PPT-pictures), and one requires semantic but not object-recognition abilities (PPT-words). All five variables were tested for multivariate normality. Two variables (WPMatch and VOSP) deviated significantly from normal and were submitted to an arcsin transformation, bringing them well within accepted standards, WP-Match: $W(18)=0.95, p<.37$, VOSP: $W(18)=0.96, p<.56$.

Data from the 20 participants were then fitted to each of the three models illustrated in Figure 6, using AMOS, a standard structural equation modelling software package. Each model in the figure depicts an alternative hypothesis about the factors that underlie the observed variation and covariation of the five measures. Latent factors are represented with ovals, and observed variables are represented with 

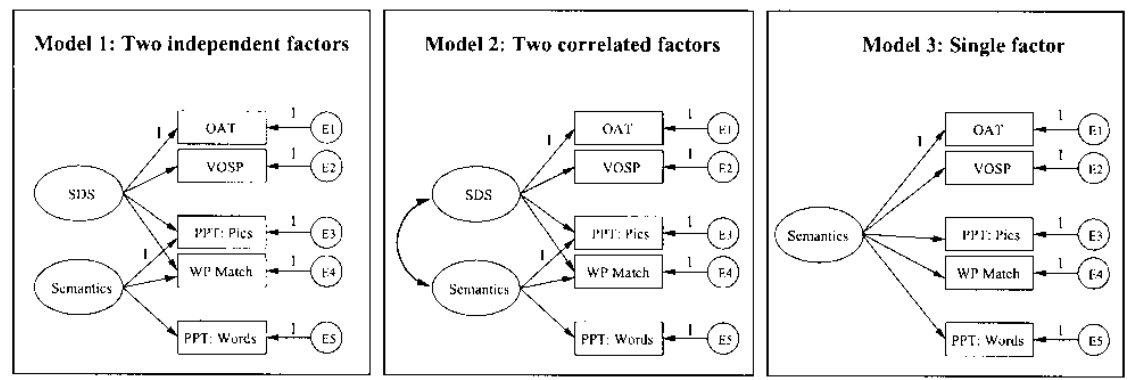

Figure 6. Structural equation models for three confirmatory factor analyses, comparing three different hypotheses about the factors underlying performance on object-decision and semantic tasks in semantic dementia.

boxes. Single-headed arrows represent the causal relations among latent and observed variables stipulated by the hypothesis in question, whereas double-headed arrows indicate that the factors they connect may be correlated. Circles labelled with an " $E$ " indicate sources of variation in the observed variables not accounted for by the latent factors in the modelfor example, measurement error, or other influences not represented in the model. Finally, numbers indicate parameters of the model that are fixed to a constant value in advance (i.e., parameters that are not estimated from the observed data). The model-fitting procedure requires that one of the free parameters associated with each latent variable (either the variance, or one of the factor loadings) be fixed. We have fixed one of the factor loadings for each latent variable to unity as is the standard custom.

With these conventions in mind it is possible to interpret the hypotheses embodied by each of the three models in our comparison. Model 1 posits that variation in the observed measure arises from two underlying factors. The first (labelled SDS for "structural description system") is the sole contributor (apart from the error term) to the object-decision tasks, and it also contributes to the two semantic tasks that require object recognition (WP-Match and PPT-pictures). The second factor (labelled Semantics) contributes to all of the semantic tasks, but not to the object-decision tasks. The absence of a double-headed arrow between Semantics and SDS indicates that, in this model, the two factors are constrained to be uncorrelated. We know from the previous analyses that this is unlikely to be a good model - the hypothesised underlying systems, even if they are functionally independent, must be conjointly impaired (i.e., correlated in the model) in order to explain the observed correlations between objectdecision performance and semantic-task performance. However we include this model specification in the current comparisons in order to determine whether we have the statistical power to reject what we know, a priori, to be a bad model of the data. 
Model 2 is almost identical to Model 1, but it includes an additional parameter: it allows Semantics and the SDS to be correlated (as indicated by the double-headed arrow). This model thus corresponds to the alternative hypothesis outlined above, that object-decision and semantic tasks are subserved by two functionally independent systems, which are conjointly impaired in semantic dementia and therefore might be correlated. Fitting of this model will allow us to determine whether we have the statistical means to differentiate this plausible model from the implausible Model 1; and to estimate just how correlated the deterioration of Semantics and the SDS must be to achieve optimal explanation of the observed data.

Model 3 represents our hypothesis about the factors underlying object decision and semantic task performance in semantic dementia. Here, all observed variation and covariation is explained with reference to a single underlying factor. A comparison of this model with Model 2 allows us to determine whether it is necessary (or preferable) to invoke two functionally independent but correlated factors to explain the observed data.

Models 1 and 3 may be viewed as more constrained hypotheses falling within the space of possibilities represented by Model 2. Model 2 treats the correlation between the two latent factors, as well as their variances, as free parameters to be estimated from the data. Model 1 differs from Model 2 only in that it fixes one of these free parameters, constraining the correlation between the two underlying factors to 0 . Model 3 differs from Model 2 by treating the two underlying factors as identical-which is equivalent to fixing their correlations to 1 , and constraining their variances to be equal. Thus Models 1 and 3 may be viewed as more specific sub-cases of Model 2-that is, they are nested within Model 2. The difference in degree of fit for two nested models is distributed as $\chi^{2}$, with degrees of freedom equivalent to the difference in degrees of freedom in the models being compared. Hence, it is easy to compare the goodness-of-fit for nested models.

Each of the three models shown in Figure 6 was fitted to the data from the five measures of interest. Two cells of data were missing; hence the models were fitted using the maximum-likelihood algorithm as required by AMOS. All models converged in less than 30 iterations.

\section{Results}

Model fit indices are given in Table 2. The $\chi^{2}$ statistic reflects the discrepancy between observed and model-predicted covariance matrices, with high values indicating a poor fit. The $p$ values associated with these indicate the probability with which the model should be rejected as a 
TABLE 2

Fits for three models of the factors underlying performance on object-decision and semantic tasks

\begin{tabular}{lrrr}
\hline Fit Stat & Model 1 & Model 2 & Model 3 \\
\hline$\chi^{2}$ & 17.0 & 0.091 & 2.49 \\
df & 3 & 2 & 5 \\
$p$ & 0.001 & 0.95 & 0.77 \\
Relative Fit & 0.761 & 0.998 & 0.979 \\
$\begin{array}{l}\text { Index (RFI) } \\
\text { Comparative Fit }\end{array}$ & 0.959 & 1.00 & 1.00 \\
$\begin{array}{l}\text { Index (CFI) } \\
\begin{array}{l}\text { Parsimony- } \\
\text { adjusted CFI }\end{array}\end{array}$ & 0.192 & 0.133 & 0.33 \\
$\begin{array}{l}\text { Akaike Information } \\
\text { Criterion (AIC) }\end{array}$ & 51 & 36 & 32 \\
\hline
\end{tabular}

possible explanation of the data. Reassuringly, Model 1 is rejected with likelihood $p<.001$, demonstrating that even with our relatively small sample size there is sufficient power to reject an inappropriate model. Neither Model 2 nor Model 3 is near to being rejected; however it is important to exercise caution in interpreting such a null result-the likelihood of failing to reject an inappropriate model is much greater when sample sizes are small.

The Relative Fit Index (RFI) reflects the normalised ratio of the model $\chi^{2}$ to that of a null-hypothesis-model in which all parameters are fixed to zero, adjusted for discrepancies in degrees of freedom. This measure varies considerably with sample size. The Comparative Fit Index (CFI) is more robust to such variation. Statistical rules-of-thumb dictate that a model should be rejected if either measure falls below 0.95 . On these grounds, Model 1 is again rejected, and Models 2 and 3 remain viable.

The parsimony-adjusted CFI and the Akaike Information Criterion (AIC) are measures that penalise models with more free parameters. Models with a higher adjusted CFI or a lower AIC may be understood to provide a more parsimonious account of the data. Model 3 performs slightly better than Model 2 on these measures.

Because Model 3 is nested within Model 2, it is possible to test the null hypothesis that the two models are identical by calculating the difference in the model $\chi^{2}$ fits, and testing this againt a $\chi^{2}$ distribution on 3 degrees of freedom (i.e., df for Model 3 - df for Model 2). A difference of 7.82 or greater is necessary to reject the null hypothesis with $p<.05$. The observed difference of $\chi^{2}=2.40$ does not exceed this criterion; hence we cannot reject the null hypothesis that the estimated best fit for Model 2 is 
identical with the more constrained hypothesis represented by Model 3. In other words, the best fit to Model 2 given the data is one in which the latent factors correlate perfectly and have the same variance-a model in which they are treated as the same factor.

\section{Discussion}

The results of Experiment 3 are only suggestive. Analysis 1 illustrated that the correlations within various object decision and semantic tasks are no greater than the correlations between these task types; and that these associations are not due to a global cognitive impairment. These results are qualitative-the relatively small size of our sample precluded strict comparison of all pairs of correlation coefficients. Analysis 2 provided a more quantitative examination of three hypotheses regarding the factors underlying performance on the various tasks, but the small sample size remains a concern here as well. In particular, low sample sizes inflate the likelihood of a Type II error: failure to reject a poor model. It is reassuring in this regard that we were able to reject a model known a priori to be unlikely. Nevertheless we cannot confidently determine, without considerably more cases, whether the two theoretically interesting models provide equally good accounts of the data.

With these caveats in mind, we would like to make two points. First, our analyses provide no evidence to support the rival hypothesis, that object recognition and semantic tasks draw upon functionally independent systems that are conjointly impaired in semantic dementia. Qualitatively, there was no indication that within-task correlations are higher than between-task correlations-indeed, the highest correlation observed was between an object-decision task (the BORB) and a verbal semantic task (the word version of the Camel and Cactus Test). The comparative factor analysis indicated that, given our limited data set, the best fit for a model with two covarying latent factors is one in which the two factors are effectively collapsed into one. Although interpretation of these results must remain tentative pending a larger sample, the suggestion they offer up is that performance on object-decision and semantic tasks in semantic dementia is governed by a single underlying factor.

Second, the two lowest correlations observed in Analysis 1 were among different object-decision tasks (specifically, the correlation of the real $>$ nonreal OAT with the BORB and with the nonreal $>$ real OAT). In light of Experiments 1 and 2, this is not very surprising-those results demonstrated that even very severe patients can perform well on the real $>$ nonreal condition of the OAT. The finding of relatively low correlations between different varieties of object decision is also consistent with recent work described by Hovius et al. (2003), which revealed that 
object-decision scores can vary considerably under semantic impairment depending upon the particular measure chosen. We believe that our account provides an explanation of this variability across task type. If object-decision and semantic task performance draw upon the same resources, then the same factors that compromise semantic task performance should also affect object-decision. In the current work we have seen that, just as patients with semantic dementia tend to retain knowledge about the typical and shared properties of objects in a domain, so too do they tend to endorse drawings of animals that have many shared and typical animal parts-regardless of whether these are real. From this we may conclude that two measures of object-decision will not correlate well if the stimulus items they employ differ considerably in typicality. More generally, the current results suggest that the variation of performance on object-decision tasks under semantic impairment is best explained with appeal to the typicality structure of the test stimulus items, rather than to the degree of impairment in a functionally independent visual recognition system.

\section{GENERAL DISCUSSION}

We opened the paper by differentiating two common hypotheses about the nature of semantic knowledge. The first, which we have described as a content-based approach, distinguishes between two qualitatively different forms of representation: semantic representations, which encode explicit semantic content; and structural representations, which support stimulus recognition but do not capture semantic content. Such a view implies that semantic content-meanings - consist in something other than knowledge about familiar objects' shapes, familiar word forms, and other surface properties, and the links among them. The second hypothesis, which we have termed a process-based approach, suggests that meanings emerge from the interactions of such surface representations in different modalities. On this view, semantic memory is best defined with reference to its function: to capture associations between the various modalityspecific surface representations that encode explicit content, such that experiencing a concept in one modality can activate its corresponding representations in other modalities.

One of the primary sources of evidence for content-based theories of semantics stems from case studies of patients who, despite impaired access to meaning from visually presented objects, appear to succeed at objectdecision tasks (e.g., Riddoch \& Humphreys, 1987). Such findings seem to suggest that the representations and processes that support visual object recognition do not depend upon intact communication with semanticsand therefore that these representations and processes are not constituents 
of the system of knowledge that encodes meanings, but are functionally separable from it. Process-based theories of semantics are challenged to explain how, if visual and semantic representations and processes are mutually interdependent, semantic impairment can possibly spare visual object recognition.

In the current paper, we have addressed this challenge by suggesting that good performance on object-decision tasks can still obtain under semantic impairment if, and only if, targets in the task respect the surface structure of the domain and distractors do not. When chimeric distractors are unusual and real-animal targets are typical-looking, patients perform well even in the face of severe semantic impairment. When real-animal targets are unusual and chimeric distractors are typical-looking, the same patients show a degree of impairment approximately commensurate with the magnitude of their semantic deficits. These results were observed both in a novel two-alternative forced-choice test of object decision, and in the more usual object-decision paradigm on a subset of stimuli from the standard test that we selected as fulfilling these typicality characteristics.

We suggest that the observed deficits are a consequence of a general amodal semantic impairment in semantic dementia, which arises with damage to the brain regions that mediate interactions among high-level perceptual representations in different modalities, as suggested by processbased theories of semantic memory. There are three observations from the present data we would like to offer in support of this hypothesis.

First, the object-decision deficits that we have chronicled parallel the general pattern of impairment observed previously in semantic dementia, in a broad range of different semantic tasks including drawing and copying (Bozeat et al., 2003; Lambon Ralph \& Howard, 2000), word-picture matching (Rogers et al., in press), and even purely verbal tasks such as attribute verification (Warrington, 1975), definition of concept names (Lambon Ralph, Graham, Patterson, \& Hodges, 1999), and word sorting (Hodges et al., 1995). All of these tasks suggest that the degradation of semantic memory in semantic dementia entails the dissolution of knowledge about the detailed properties of objects that differentiate them from their semantic neighbours, with relative sparing of knowledge about properties that are generally characteristic of objects in the domain. As we have seen, object decision is no different: participants endorse items with many typical and few idiosyncratic visual properties, and reject items composed primarily of atypical or unusual properties. This influence of typicality was observed in both the novel test described in Experiment 1 and a subset of items from the familiar BORB object-decision test (Experiment 2).

Second, the degree of impairment in the nonreal $>$ real condition of the OAT correlates strongly with the overall degree of semantic impairment, 
as does performance in the BORB object-recognition task (see Hovius et al., 2003, as well as the current results). Indeed, object-decision performance on various different versions of the task correlated as well with semantic tasks as with one another (Experiment 3 ). The symmetry of the current findings with past results in other semantic tasks, coupled with the finding of generally high correlations between object-decision and semantic tasks, together suggest that the system supporting visual object recognition is part and parcel of the general system of semantic knowledge that deteriorates in semantic dementia.

Third, the factor analysis described in Experiment 3 revealed that the best-fitting parameterisation of a two-factor model explaining the observed covariances among two object-decision and three semantic tasks is one in which the two latent factors are effectively collapsed into one. Although this result is not conclusive given the small sample size, the analysis does not compel us to invoke more than a single underlying factor to explain performance on these different tasks.

Thus the present data are at least consistent with a theory of semantic memory in which semantic knowledge emerges from the interactive activation of perceptual representations in various sensory and verbal modalities. We might further inquire whether there are other empirical or theoretical reasons for preferring a process-based theory of semantics to content-based approaches. This is of course a subject of controversy with a scope that extends beyond the neuropsychological considerations addressed here. There are, however, two points that we would like to make with respect to this question.

First, we believe that our data call into question one of the primary reasons for differentiating between "meaningful" semantic representations and "non-meaningful" structural representations. Several key researchers in the field have strongly emphasised the importance of data from both object- and word-recognition under semantic impairment for motivating this distinction (see Humphreys et al., 1988; Coltheart et al., 1998; Lauro-Grotto, Piccini, \& Shallice, 1997). We have offered an alternative explanation for the apparent sparing of object recognition under semantic impairment, which depends upon assumptions about the structure of interacting representations rather than upon claims about the functional architecture of separate recognition and semantic systems. It is almost certainly possible to explain our results within a more functionally segregated framework; but we suggest that our data begin to challenge one of the primary reasons for presupposing such segregation in the first place. Further research will be necessary to determine whether a similar account might extend to explain other puzzling phenomena, such as the apparent sparing of word-recognition under semantic impairment (e.g., Ward, Stott, \& Parkin, 2000), though preliminary results in this 
endeavour are encouraging (see Rogers, Lambon Ralph, Hodges, \& Patterson, in press).

Second, we do not believe that alternative approaches that have heretofore differentiated meaningful from structural representations would lose any of their appeal were they to discard the distinction. Our reasoning derives from a consideration of one of the best-known neuropsychological models of naming from vision-the interactiveactivation (IAC) model proposed by Humphreys and colleagues (Humphreys et al., 1988, 1995)-and how it has evolved in recent years (Humphreys \& Forde, 2001).

The IAC model consists of three processing layers that locally represent different kinds of information. Structural descriptions are coded in one layer, knowledge about the functional or associative semantic properties of objects is stored in a second layer, and lexical/phonological representations of known words are stored in a third. Visual stimuli excite stored structural descriptions of known objects in parallel, in proportion to the degree of structural overlap between the stimulus and the stored representations. Activation then cascades forward to semantic representations, which explicitly encode information about the functional and associative properties of objects, and from there to lexical or phonological representations of words.

In its original formulation (Humphreys et al., 1988), the IAC model included only feed-forward connections from structural descriptions to semantics. Because activity in the semantic system did not feed back to influence activity in the structural description system, disruption of communication between structural descriptions and semantics had no consequence for the activation of the structural descriptions themselves. The model's ability to explain data from patients like J.B. (who showed poor access to semantic information from vision, but relatively good object-decision performance) thus relied upon what amounts to a theoretical claim about the functional independence of visual object recognition and semantic processes.

As originally described, the IAC model exemplifies a content-based approach to semantics. The representations that encode visual structural information are cast as being separate from those encode semantic content; and the processes that support visual recognition and semantic memory, far from being mutually interdependent, are posited to be functionally independent.

Later instantiations of the theory, including an implemented computational model described by Humphreys et al. (1995), altered the original design by incorporating feedback connections from semantic to structural description representations. Semantic representations were still cast as encoding a qualitatively different kind of information than structural 
descriptions but, more in line with process-based approaches, the two systems were described as interacting. This alteration did not compromise the model's ability to explain the time-course of processing in various kinds of semantic tasks, or other appealing aspects of the theory (Humphreys \& Forde, 2001). Not surprisingly, however, it did compromise the functional independence of structural descriptions and semantics. Because the states of semantic units in this implementation can influence the activation of structural descriptions, one would expect degraded semantic processing to have consequences for the resolution of competition at the level of structural descriptions (and, therefore, for object recognition).

In the most recent incarnation of the IAC approach (the HIT model; see Humphreys \& Forde, 2001), the authors propose that each modality of perception contains its own perceptual recognition stores, akin to the structural description system in vision, which interact with one another either directly or via Damasio's "convergence zones". Here, separate semantic representations that code the meanings of words and objects have effectively disappeared from the picture-the retrieval of information about objects arises from the interaction of these different perceptual knowledge stores. The theory is, in fact, just the process-based approach to semantics that we have been advocating in the present work; and as the authors note, it obviates the notion that semantic knowledge resides in a separate semantic store (with the possible exception of "encyclopaedic" information about objects).

In moving away from functionally separable structural and semantic stores, and toward a more interactive and process-based approach, the HIT framework raises the same question that the IAC model was intended to address: how are patterns of impairment such as J.B.'s possible? If semantic knowledge arises simply from the interactive activation of modality-specific representations, why should the degradation of these interactions spare visual object recognition? This is, after all, the issue that prompted claims of functional separability between meaningful and structural representations in the first place.

The current work allows us to see how apparent dissociations of object recognition and semantic knowledge might arise as a consequence of representational structure in a recurrent, interactive and distributed system, such as the one we have described elsewhere (Rogers et al., in press). Deficits such as J.B.'s do not lay bare the functional separability of two independent systems, but simply reflect sensitivity to the same factors that govern impaired performance in all semantic tasks. Indeed, one version of the HIT framework described by Humphreys and Forde (2001) - the version that incorporates convergence zones (see Figure 4 in that paper) - is similar in many respects to a computational model we have 
used to explain a range of phenomena in semantic dementia (Rogers, Lambon-Ralph, Patterson, McClelland, \& Hodges, 1999). We view our model as similar in spirit (if different in some of the details) to this version of the HIT framework; and the family of distributed and interactive semantic models as alternative implementations of a similar, process-based theory of semantics.

\section{REFERENCES}

Allport, D.A. (1985). Distributed memory, modular systems and dysphasia. In S.K. Newman \& R. Epstein (Eds.), Current perspectives in dysphasia. Edinburgh: Churchill Livingstone.

Barbarotto, R., Capitani, E., Spinnler, H., \& Trivelli, C. (1995). Slowly progressive semantic impairment with category specificity. Neurocase, 1, 107-119.

Barsalou, L.W. (this issue). Situated simulation in the human conceptual system. Language and Cognitive Processes, 18, 513-562.

Biederman, I. (1987). Recognition-by-components: A theory of human image understanding. Psychological Review, 94, 115-147.

Bozeat, S., Lambon Ralph, M.A., Graham, K.S., Patterson, K.,Wilkin, H., Rowland, J., Rogers, T., \& Hodges, J.R. (2003). A duck with four legs: Investigating the structure of conceptual knowledge using picture drawing in semantic dementia. Cognitive Neuropsychology, 20, 27-47.

Bozeat, S., Lambon Ralph, M.A., Patterson, K., Garrard, P., \& Hodges, J.R. (2000). Nonverbal semantic impairment in semantic dementia. Neuropsychologia, 38, 1207-1215.

Bozeat, S., Lambon Ralph, M.A., Patterson, K., \& Hodges, J.R. (in press). When objects lose their meaning: what happens to their use? Cognitive, Affective, and Behavioral Neuroscience.

Bryant, F.B., \& Yarnold, P.R. (1995). Principal components analysis and exploratory and confirmatory factor analysis. In P.R. Grimm \& L.G. Grimm (Eds.), Reading and understanding multivariate analysis. Washington, DC: American Psychological Association Books.

Chao, L.L., Haxby, J.V., \& Martin, A. (1999). Attribute-based neural substrates in temporal cortex for perceiving and knowing about objects. Nature Neuroscience, 2, 913-919.

Coltheart, M., Inglis, L., Michie, P., Bates, A., \& Budd, B. (1998). A semantic subsystem of visual attributes. Neurocase, 4, 353-370.

Coltheart, M., Rastle, K., Perry, C., Langdon, R., \& Ziegler, J. (2001). DRC: A dual route cascaded model of visual word recognition and reading aloud. Psychological Review, 108, 204-256.

Cree, G., \& McRae, K. (2002). Factors underlying category-specific deficits. In E.M.E. Forde \& G.W. Humphreys (Eds.), Category specificity in brain and mind. Hove, UK: Psychology Press.

Damasio, H., Grabowski, T.J., Tranel, D., \& Hichwa, R.D. (1996). A neural basis for lexical retrieval. Nature, 380, 499-505.

Eggert, G.H. (1977). Wernicke's works on aphasia: A sourcebook and review (Vol. 1). The Hague: Mouton.

Funnell, E. (1996). Response biases in oral reading: An account of the co-occurrence of surface dyslexia and semantic dementia. Quarterly Journal of Experimental Psychology, $49 A, 417-446$.

Garrard, P., \& Hodges, J.R. (2000). Semantic dementia: Clinical, radiological, and pathological perspectives. Journal of Neurology, 247, 409-422. 
Garrard, P., Lambon Ralph, M., \& Hodges, J.R. (2002). Semantic dementia: A categoryspecific paradox. In E.M.E. Forde \& G.W. Humphreys (Eds.), Category specificity in brain and mind (pp. 149-179). Hove, UK: Psychology Press.

Garrard, P., Lambon Ralph, M.A., Hodges, J.R., \& Patterson, K. (2001). Prototypicality, distinctiveness and intercorrelation: Analyses of the semantic attributes of living and nonliving concepts. Cognitive Neuroscience, 18, 125-174.

Graham, K.S., Simons, J.S., Pratt, K.H., Patterson, K., \& Hodges, J.R. (2000). Insights from semantic dementia on the relationship between episodic and semantic memory. Neuropsychologia, 38, 313-324.

Hillis, A.E., \& Caramazza, A. (1995). Cognitive and neural mechanisms underlying visual and semantic processing: Implications from "optic aphasia". Journal of Cognitive Neuroscience, 7, 457-478.

Hodges, J.R., Bozeat, S., Lambon Ralph, M.A., Patterson, K., \& Spatt, J. (2000). The role of conceptual knowledge in object use: Evidence from semantic dementia. Brain, 123, 19131925.

Hodges, J.R., Garrard, P., \& Patterson, K. (1998). Semantic dementia and pick complex. In A. Kertesz \& D. Munoz (Eds.), Pick's disease and pick complex. New York: Wiley Liss.

Hodges, J.R., Garrard, P., Perry, R., Patterson, K., Bak, T., \& Gregory, C. (1999a). The differentiation of semantic dementia and frontal lobe dementia from early alzheimer's disease: A comparative neuropsychological study. Neuropsychology, 13, 31-40.

Hodges, J.R., Graham, N., \& Patterson, K. (1995). Charting the progression in semantic dementia: Implications for the organisation of semantic memory. Memory, 3, 463-495.

Hodges, J.R., Patterson, K., \& Tyler, L.K. (1994). Loss of semantic memory: Implications for the modularity of mind. Cognitive Neuropsychology, 11, 505-542.

Hodges, J.R., Spatt, J., \& Patterson, K. (1999b). What and how: Evidence for the dissociation of object knowledge and mechanical problem-solving skills in the human brain. Proceedings of the National Academy of Sciences, 96, 9444-9448.

Hovius, M., Kellenbach, M., Graham, K.S., Hodges, J., \& Patterson, K. (2003). What do tests of object decision measure? Evidence from semantic dementia. Neuropsychology, 17, 100-107.

Howard, D., \& Patterson, K. (1992). Pyramids and palm trees: A test of semantic access from pictures and words. Bury St. Edmunds, UK: Thames Valley.

Humphreys, G.W., \& Forde, E.M.E. (2001). Hierarchies, similarity, and interactivity in object-recognition: On the multiplicity of 'category-specific' deficits in neuropsychological populations. Behavioral and Brain Sciences, 24, 453-509.

Humphreys, G., Lamote, C., \& Lloyd-Jones, T.J. (1995). An interactive activation approach to object processing: Effects of structural similarity, name frequency, and task in normality and pathology. Memory, 3, 535-586.

Humphreys, G.W., \& Riddoch, M.J. (1987). To see but not to see: A case-study of visual agnosia. Hove, UK: Lawrence Erlbaum Associates Ltd.

Humphreys, G.W., \& Riddoch, M.J. (1993). Interactions between object and space systems revealed through neuropsychology. In D.E. Meyer \& S. Kornblum (Eds.), Attention and Performance XIV: Synergies in experimental psychology, artifical intelligence, and cognitive neuroscience. Hillsdale, NJ: Lawrence Erlbaum Associates, Inc.

Humphreys, G.W., \& Riddoch, M.J. (1999). Impaired development of semantic memory: Separating semantic from structural knowledge and diagnosing a role for action in establishing stored memories for objects. Neurocase, 5, 519-532.

Humphreys, G.W., Riddoch, M.J., \& Quinlan, P.T. (1988). Cascade processes in picture identification. Cognitive Neuropsychology, 5, 67-103. 
Kellenbach, M., Brett, M., \& Patterson, K. (2001). Large, colorful or noisy? Attribute- and modality-specific activations during retrieval of perceptual attribute knowledge. Cognitive, Affective and Behavioral Neuroscience, 1, 207-221.

Lambon Ralph, M., Graham, K.S., Patterson, K., \& Hodges, J.R. (1999). Is a picture worth a thousand words? Evidence from concept definitions by patients with semantic dementia. Brain and Language, 70, 309-335.

Lambon Ralph, M.A., \& Howard, D. (2000). Gogi-aphasia or semantic dementia? Simulating and assessing poor verbal comprehension in a case of progressive fluent aphasia. Cognitive Neuropsychology, 17, 437-465.

Lambon Ralph, M.A., McClelland, J., Patterson, K., Galton, C.J., \& Hodges, J. (2001). No right to speak? The relationship between object naming and semantic impairment: Neuropsychological evidence and a computational model. Journal of Cognitive Neuroscience.

Lauro-Grotto, R., Piccini, C., \& Shallice, T. (1997). Modality-specific operations in semantic dementia. Cortex, 33, 593-622.

Lissauer, H. (1890). Ein fall von seelenblindheit nebst einem beitrage zur theorie derselben. Archiv fur Psychiatrie und Nervenkrankheit, 21, 222-270.

Marr, D. (1982). Vision. San Francisco, CA: W.H. Freeman.

Patterson, K., \& Hodges, J. (2000). Semantic dementia: One window on the structure and organisation of semantic memory. In F. Boller \& J. Grafman (Eds.), Handbook of neuropsychology: Vol. 2. Memory and its disorders (2nd edn., pp. 313-333). Amsterdam: Elsevier Science.

Perry, R.J., \& Hodges, J.R. (1999). Attention and executive deficits in Alzheimer's disease: A critical review. Brain, 122, 383-404.

Plaut, D.C. (2002). Graded modality-specific specialisation in semantics: A computational account of optic aphasia. Cognitive Neuropsychology, 19, 603-639.

Pulvermuller, F. (1999). Words in the brain's language. Behavioral and Brain Sciences, 22, 253-336.

Quinn, P., Johnson, M.H., Mareschal, D., Rakison, D., \& Younger, B.A. (2000). Understanding early categorization: One process or two? Infancy, 1, 111-122.

Riddoch, M.J., \& Humphreys, G.W. (1987). Visual object processing in optic aphasia: A case of semantic access agnosia. Cognitive Neuropsychology, 4, 131-185.

Riddoch, M.J., \& Humphreys, G.W. (1993). BORB: Birmingham Object Recognition Battery. Hove, UK: Psychology Press.

Rogers, T.T., Lambon-Ralph, M., Garrard, P., Bozeat, S., McClelland, J.L., Hodges, J.R., \& Patterson, K. (in press). The structure and deterioration of semantic memory: A computational and neuropsychological investigation. Psychological Review.

Rogers, T.T., Lambon-Ralph, M., Hodges, J.R., \& Patterson, K. (in press). Natural selection: The impact of semantic impairment on lexical and object decision. Cognitive Neuropsychology.

Rogers, T.T., Lambon-Ralph, M., Patterson, K., McClelland, J.L., \& Hodges, J.R. (1999). A recurrent connectionist model of semantic dementia. In Cognitive neuroscience society annual meeting program 1999. Retrieved from http://cognet.mit.edu/posters/ poster.tcl?publication_id=3664

Rogers, T.T., \& McClelland, J.L. (in press). Semantic cognition: A parallel distributed processing approach. Cambridge, MA: MIT Press.

Rogers, T.T., \& Plaut, D.C. (2002). Connectionist perspectives on category specific deficits. In E. Forde \& G. Humphreys (Eds.), Category specificity in mind and brain. Hove, UK: Psychology Press.

Rumiati, R., \& Humphreys, G.W. (1997). Visual object agnosia without alexia or prosopagnosia: Arguments for separate knowledge stores. Visual Cognition, 4, 207-217. 
Sheridan, J., \& Humphreys, G.W. (1993). A verbal-semantic category-specific recognition impairment. Cognitive Neuropsychology, 10, 185-200.

Smith, L.B. (2000). From knowledge to knowing: Real progress in the study of infant categorization. Infancy, 1, 91-97.

Snowden, J.S., Goulding, P.J., \& Neary, D. (1989). Semantic dementia: A form of circumscribed cerebral atrophy. Behavioral Neurology, 2, 167-182.

Stewart, F., Parkin, A.J., \& Hunkin, N.M. (1992). Naming impairments following recovery from herpes simplex encephalitis: Category specific? Quarterly Journal of Experimental Psychology, 44A, 261-284.

Tyler, L., Moss, H.E., Durrant-Peatfield, M.R., \& Levy, J. P. (2000). Conceptual structure and the structure of concepts: A distributed account of category-specific deficits. Brain and Language, 75, 195-231.

Ward, J., Stott, R., \& Parkin, A.J. (2000). The role of semantics in reading and spelling: Evidence for the 'summation hypothesis'. Neuropsychologia, 38, 1643-1653.

Warrington, E.K. (1975). The selective impairment of semantic memory. Quarterly Journal of Experimental Psychology, 27, 635-657.

Warrington, E.K., \& James, M. (1991). Visual Object and Space Perception battery. Bury St. Edmunds, UK: Thames Valley Test Company.

Warrington, E.K., \& McCarthy, R. (1987). Categories of knowledge: Further fractionation and an attempted integration. Brain, 110, 1273-1296. 